\title{
Biologically-inspired time and location of impact prediction from optical flow
}

\author{
Chris McCarthy* \\ * NICTA Canberra Research Lab \\ Canberra, Australia \\ and \\ *Australian National University \\ College of Engeering and Computer Science \\ Canberra, Australia \\ chris.mccarthy@nicta.com.au
}

\author{
Giorgio Metta ${ }^{\dagger}$ \\ †Italian Institute of Technology \\ Genova, Italy \\ giorgio.metta@iit.it
}

\begin{abstract}
We investigated the use of optical flow to predict the time and location of impact of an incoming object. By examining local patterns of optical flow, we make predictions on an object's trajectory with respect to a stationary observer, and its time-to-contact with the observer's (assumed planar) body. Such a cue may serve as inputs to motor responses for reactive threat avoidance, or object interception (such as catching a ball). The presented approach is based on the observed behaviour of neurons in the $\mathrm{F} 4$ region of the pre-motor cortex of primates and it is part of a larger project which aims at modelling multi-sensory neurons and their contribution to reaching behaviour on the iCub humanoid platform. We present preliminary experimental results of a computation model of F4's visual responses using real image sequences acquired from the robot, and demonstrate their application in a real-time threat detection/prediction system.
\end{abstract}

Keywords-optical flow, time-to-contact, cognitive robotics

\section{INTRODUCTION}

An important capability of any system (artificial or biological) working in a dynamic environment is the ability to perceive independently moving objects. This is particularly important when the object poses an imminent threat of collision with the observer. Detection of the threat must occur with sufficient time to allow evasive actions to be taken. This may involve movement away from the threat to avoid collision, or the invocation of motor responses to intercept its course (e.g., catching or deflecting an object). In either case, the ability to detect a potential threat and its likely trajectory, is essential.

The primate visual system is well equipped for such tasks. Indeed, neuroscience researchers have identified specific neurons in the pre-motor cortex of primates (area F4) responsible for the sensory guidance of reaching and intercepting/avoiding incoming objects [1]. One influential hypothesis poses that space is not coded unitarily in the brain (e.g., as a Cartesian coordinate system) but rather it is effector-based, and therefore the neural coding depends on the goal (attention, reaching, grasping) [2]. Multiple fronto-parietal circuits are responsible for the sensory to motor transformations required for moving in space in a complex interplay between forward and backward neural projections. The neurons of area F4 in particular are multisensory showing overlapping visual and tactile receptive fields. Their motor responses are consistent with a role in the control of reaching movements. Further, it has been shown that each receptive field is connected to a specific body part irrespective of the posture and eye/body relative configuration [3]. Later, Graziano et al. [4] have shown how F4 responses code for the position of the hand in space and therefore form an important component of the planning of reaching.

The visual response of F4 neurons has been interpreted as a prediction of the impending impact of a moving object into a given body part; a sort of extension of the tactile receptive field in 3D space. The receptive field size in 3D depends on the speed of the object and therefore it is natural to think their responses as information related to the estimated time to impact as they can be derived from visually estimated velocity. Thus, it is of interest to consider the use of optical flow as a primary cue for predicting the course of incoming objects.

In computer vision, the traditional approach to object motion estimation has been to explicitly estimate a motion model for surfaces in the scene. Geometric methods such as [5], [6], for example, attempt to optimally recover the motion of all objects in the scene from matched points in two or more views. While often highly accurate, these techniques are not feasible for real-time use. Other approaches examine the apparent motion of objects in the scene via explicit tracking of feature points [7], [8], or via the optical flow field generated by the motion of objects [9], [10]. While providing highly accurate motion models, such techniques are not suitable for eliciting reflex responses to incoming objects.

An alternative approach is to use more direct visual cues that are measurable from the optical flow. The relative motion of a surface towards an observer induces an apparent expansion, or looming effect in the observer's image. Under certain constraints, the measure of this flow field divergence directly relates surface distance and relative velocity towards the observer, thus defining the time-to-impact $(\tau)$.

The robust estimation of $\tau$ from optical flow has been a topic of interest in biological and computer/robot vision for some time [11], [12]. In robotics, $\tau$ is most commonly used 
for obstacle avoidance [12]-[14]. More recently it has been used to support docking [15], and landing [16] manoeuvres. Such approaches provide a direct means of estimating the rate of approach of objects without explicitly computing the object's motion parameters. Such work, however, provides an estimation of $\tau$ with respect to the camera only. In the context of an embodied system seeking to avoid impact with an incoming object, an estimate of $\tau$ with respect to its predicted location of impact is significantly more useful.

In this paper we seek to extend the use of flow-based $\tau$ estimation to the task of predicting where, and at what time, impact will occur with respect to a virtual plane about the camera. To obtain fast impact predictions, and to maintain relevance to the underlying biological model, we compute time and location of impact from instantaneous local patterns of optical flow (i.e., we do not perform temporal integration). We present preliminary results over real image sequences showing that workable estimates of both time and location of impact can be obtained using directly measured visual quantities. Moreover, these results are achieved in real-time, thereby demonstrating the proposed algorithm's potential application to reactive threat avoidance and interception tasks.

The paper is structured as follows. In Section II we outline the proposed method for estimating time and location of impact. Section III presents results from experimental performance evaluation. Section IV provides a discussion of the results. Finally, Section V concludes the paper and outlines future directions for this research.

\section{THEORY}

The current work aims to assess the feasibility of estimating time and location of impact from local instantaneous flow field patches. We therefore make the following assumptions: a stationary camera, predominantly translational motion of a single incoming object, and a roughly planar surface facing towards the camera. We derive the proposed technique under a spherical projection model, thus generalising the viewing direction. Note that a spherical mapping from standard perspective projection is easily obtained from camera calibration ${ }^{1}$. Before presenting the proposed technique, we briefly review time-to-contact estimation from spherical flow.

\section{A. Preliminaries: computing $\tau$ from spherical flow}

The computation of time-to-contact from local flow field divergence has been studied extensively (e.g., [12], [17], [18]). We briefly outline the derivation of time-to-contact from spherical flow field divergence and how this is applied to the current problem.

Considering a translating object with respect to a stationary camera, we define the induced optical flow on the unit view sphere as [19]:

$$
f(\hat{p})=-\frac{v}{|R(\hat{p})|}((\hat{t} \cdot \hat{p}) \hat{p}-\hat{t}),
$$

\footnotetext{
${ }^{1}$ and is not crucial if the field of view is small
}

where $\hat{p} \in \mathbb{R}^{3}$ is the direction of an arbitrary point on the sphere, $\hat{t}$ is the direction of motion of the object, $v$ its velocity, and $R(\hat{p})$ its radial distance along $\hat{p}$. We assume any rotation of the object is neglibly small with respect to its translation.

The flow field divergence of the spherical flow field (as originally derived by [17]) is:

$$
\operatorname{div}(\hat{p})=-\frac{v(\hat{p} \cdot \hat{t})}{R(\hat{p})}\left[2+\frac{\nabla R(\hat{p})}{R(\hat{p})}\left(\frac{\hat{t}}{(\hat{p} \cdot \hat{t})}-\hat{p}\right)\right],
$$

where $\nabla R(\hat{p})$ is the local depth gradient of the surface patch projecting into the tangent plane about $\hat{p}$. Re-arranging Eq. (2), we obtain the time-to-contact with respect to the camera (i.e., along the viewing direction $\hat{p}$ ):

$$
\tau_{\mathrm{p}}=\frac{-}{R(\hat{p})} v(\hat{p} \cdot \hat{t})=\frac{1}{\operatorname{div}(\hat{p})}\left[2+\frac{\nabla R(\hat{p})}{R(\hat{p})}\left(\frac{\hat{t}}{(\hat{p} \cdot \hat{t})}-\hat{p}\right)\right] .
$$

The depth gradient, $\nabla R(\hat{p})$, introduces a deformation component into the divergence estimation, denying a precise estimate of time-to-contact. However, this deformation will be small for objects sufficiently far away, and thus we assume zero depth gradient within the region of interest, reducing (3) to:

$$
\tau_{\mathrm{p}}=-\frac{R(\hat{p})}{v(\hat{p} \cdot \hat{t})}=\frac{1}{\operatorname{div}(\hat{p})} .
$$

\section{B. Proposed method}

We seek to predict the relative impact location of an incoming object on a planar body centred on the image origin. We regard the planar body, $\mathbf{B}$, to be the infinite extension of the image plane in all directions. We derive the method in the following logical steps: 1 . compute the angle of approach with respect to the viewing direction; 2. estimate location of impact with respect to the camera centre; and 3. estimate timeto-impact at the predicted impact location.

1) Computing angle of approach: We seek to estimate the angle of approach of the object with respect to the viewing direction, $\hat{p}$. This may then be used to obtain an angle of approach with respect to the normal of the planar body $\mathbf{B}$.

Let $\hat{q}$ be the direction of the object's translational motion parallel to the tangent plane about $\hat{p}$. We define the component of optical flow in this direction as:

$$
\begin{aligned}
f(\hat{p}) \cdot \hat{q} & =\frac{-v}{R(\hat{p})}((\hat{p} \cdot \hat{t})(\hat{p} \cdot \hat{q})-(\hat{t} \cdot \hat{q})) \\
& =\frac{v(\hat{t} \cdot \hat{q})}{R(\hat{p})} .
\end{aligned}
$$

To obtain the ratio of motion parallel to the tangent plane of $\hat{p}$, we multiply (5) by $\tau_{p}$ such that:

$$
\frac{f(\hat{p}) \cdot \hat{q}}{\operatorname{div}(\hat{p})}=\frac{(\hat{t} \cdot \hat{q})}{(\hat{t} \cdot \hat{p})} .
$$

The approach angle with respect to $\hat{p}\left(\theta_{p}\right)$ is then given by:

$$
\theta_{p}=\tan ^{-1}\left(\frac{(\hat{t} \cdot \hat{q})}{(\hat{t} \cdot \hat{p})}\right) .
$$




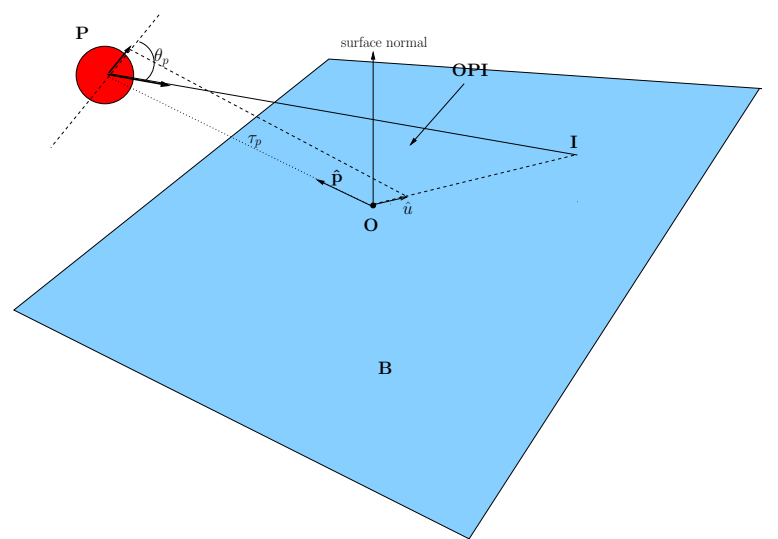

Fig. 1. Geometric representation of impact point $(I)$ prediction of an incoming object $P$, on the planar body B. $O$ is the camera-centred origin.

2) Estimating location of impact: We now compute the location of impact in B. Specifically, we compute the direction $\hat{u} \in \mathbf{B}$, and the scaled radial distance $d_{u}$ of the impact point, $I$, with respect to the centre of $\mathbf{B}$ (i.e., the origin, $O$ ). To assist these derivations, we first define the plane containing $O, P$, and the yet to be determined $I$ (see Figure 1). Note that this plane contains $\hat{t}$, and thus defines the object trajectory plane. We refer to this triangular region as OPI.

We first consider $\hat{u}$. The line defined by $\hat{u}$ in $\mathbf{B}$ is given by the intersection of OPI with $\mathbf{B}$. Thus, given knowledge of $\hat{p}$ with respect to $B$ (from camera calibration), and $\hat{q}$ from the optical flow, $\hat{u}$ may be determined by translating $\hat{q}$ along $\hat{p}$ to the origin, and projecting it into $\mathbf{B}$. This intersection defines a line of possible impact locations (see Figure 1). We refer to this line as the line of impact.

To recover $d_{u}$, it is necessary to first determine the current projected location of $P$ on the infinitely extended line of impact $(\hat{u})$. Note that $\hat{u}$ exists in both $\mathbf{B}$ and OPI, thus allowing $d_{u}$ to be recovered without regard for the relative orientation of $\mathbf{B}$ and OPI. We therefore consider only the plane of OPI in the remainder of this derivation (see Figure 2).

Let $\hat{n} \in \mathbb{R}^{2}$ define a vector orthogonal to $\hat{u}$ in $\mathbf{O P I}$, and $\phi$ be the angle between the directions $\hat{p}$ and $\hat{n}$. Let $N$ denote the current projected location of $P$ on the line of impact (i.e., the projection of $P$ along $\hat{n}$ ), and $d_{n}$ be the scaled radial distance of $N$ from the the origin. We solve for $d_{n}$ by projecting $\tau_{p}$ onto the line of impact such that:

$$
d_{n}=\tau_{p} \sin (\phi)
$$

Let $d$ be the total scaled distance between $N$ and $I$, and $\theta_{n}$ be the approach angle of the object with respect to $\hat{n}$, such that:

$$
\theta_{\mathrm{n}}=\theta_{\mathrm{p}}+\phi
$$

Projecting $\tau_{\mathrm{p}}$ along $\hat{n}$ :

$$
\tau_{\mathrm{n}}=\tau_{p} \cos \phi
$$

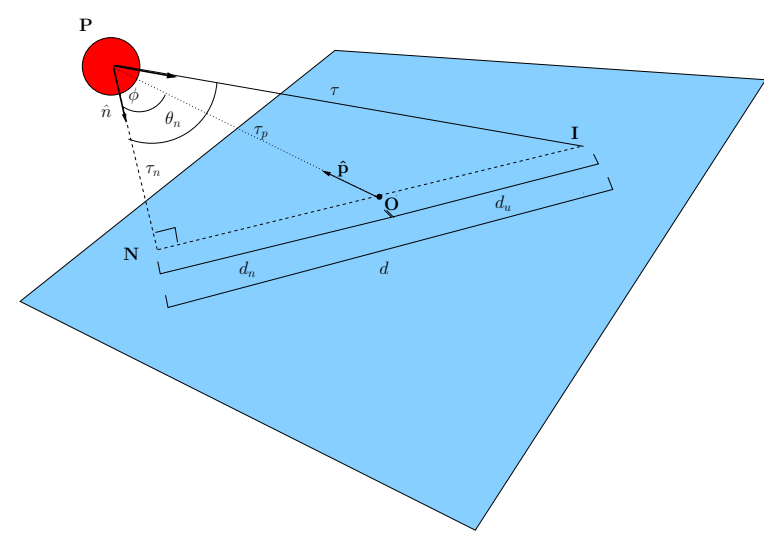

Fig. 2. Figure shows geometric relationships exploited to predict the scaled distance of the point of contact along the line of impact with respect to the origin.

we solve for $d$ such that:

$$
\begin{aligned}
d & =\tau_{\mathrm{n}} \tan \theta_{\mathrm{n}}, \\
& =\tau_{p} \cos \phi \tan \theta_{\mathrm{n}} .
\end{aligned}
$$

Given $d_{n}$ and $d$, we may determine the radial distance, $d_{u}$ :

$$
d_{u}=d-d_{n} .
$$

Expressing this in terms of directly measurable (or known) quantities:

$$
d_{u}=\tau_{\mathrm{p}}\left(\cos \phi \tan \left(\theta_{\mathrm{p}}+\phi\right)-\sin (\phi)\right) .
$$

Note that $d_{u}$ is defined as a proportion of $\tau_{\mathrm{p}}$, thereby defining the radial distance of impact in temporal units scaled by the measured time-to-contact along the viewing direction.

\section{Estimating time-to-impact with the plane}

To infer the incoming object's time-to-impact, $\tau$, with $I$ we compute the temporal distance along the angle of approach from $\tau_{\mathrm{n}}$ and $d$ such that:

$$
\tau=\sqrt{\tau_{n}^{2}+d^{2}}
$$

Substituting for $\tau_{n}$ and $d$, we obtain:

$$
\begin{aligned}
\tau & =\tau_{p} \sqrt{\cos ^{2} \phi+\left(\cos ^{2} \phi \tan ^{2}\left(\theta_{\mathrm{p}}+\phi\right)\right)}, \\
& =\tau_{p} \cos \phi \sec \left(\theta_{p}+\phi\right) .
\end{aligned}
$$

\section{Performance ASSESSMent}

\section{A. Implementation}

1) Divergent motion segmentation: The first step of the algorithm identifies regions of looming motion in the image. This is achieved using simple image differencing of image intensity values, and applying an appropriate threshold to account for noise. The optical flow within each region is then computed and used to compute the divergence, from which the region of maximum divergence is selected. Optical flow was estimated using a pyramidal version of Lucas and Kanade's 

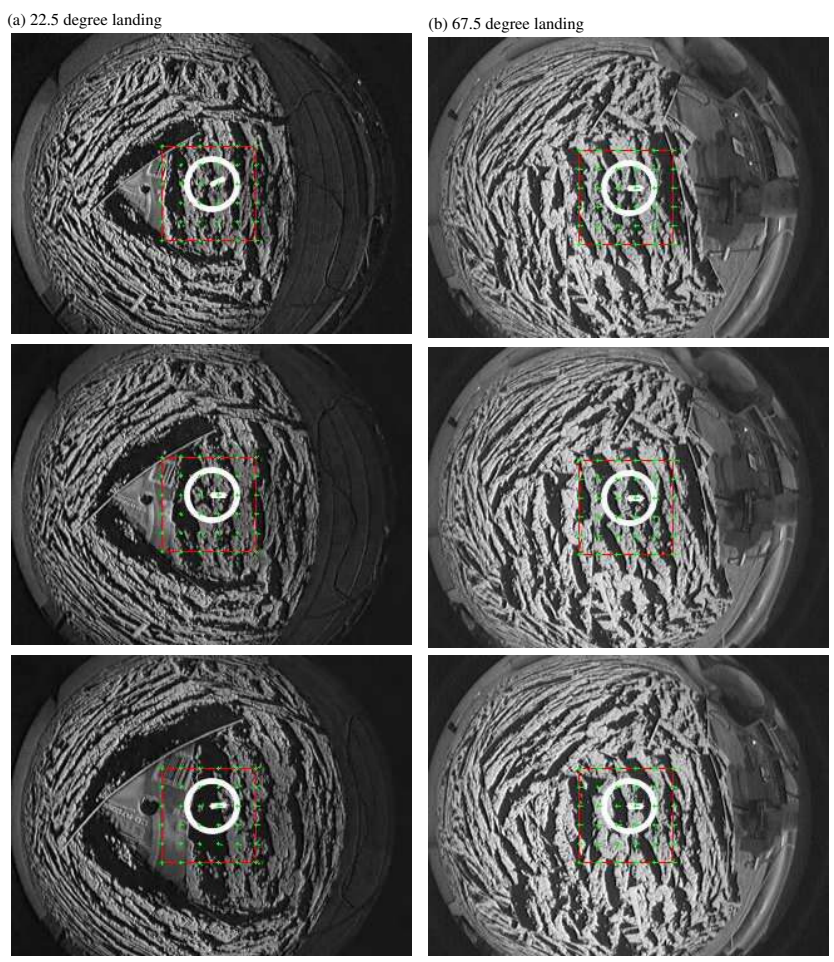

Fig. 3. Sample frames from both the $22.5^{\circ}$ and $67.5^{\circ}$ landing sequences, showing the central region for which the angle of approach, and impact location are estimated.

method [20], provided in the open source library, OpenCV ${ }^{2}$. Divergence is computed by summing the result of size five linear Sobel kernels convolved in over horizontal and vertical flow components in each respective direction. Segmentation is achieved by growing regions of motion where positive flow divergence above a set threshold exists. Thresholding is performed on the average divergence computed in $5 \times 5$ pixel regions. Neighbouring regions above the threshold are then combined.

2) Impact estimation: The approach angle is obtained by first estimating the predominant direction of motion of the object in the image plane. Taking the point of maximum divergence, eight discrete templates of unit flow vector directions are convolved over the segmented region of interest to obtain a direction of most support.

The angle of approach with respect to the viewing direction is computed by dividing the magnitude of the optical flow in the identified dominant translational direction (as given by the score), by the average of divergence estimates taken within the same region. We then compute $\theta_{\mathrm{p}}$ from this ratio (i.e., 7), from which we obtain $\theta_{n}$ via (9) and the time-to-impact via (15).

\section{B. Controlled-conditions testing}

A basic implementation of the proposed algorithm was run over two image sequences depicting the controlled motion

\footnotetext{
${ }^{2} \mathrm{http}: / /$ opencv.willowgarage.com
}

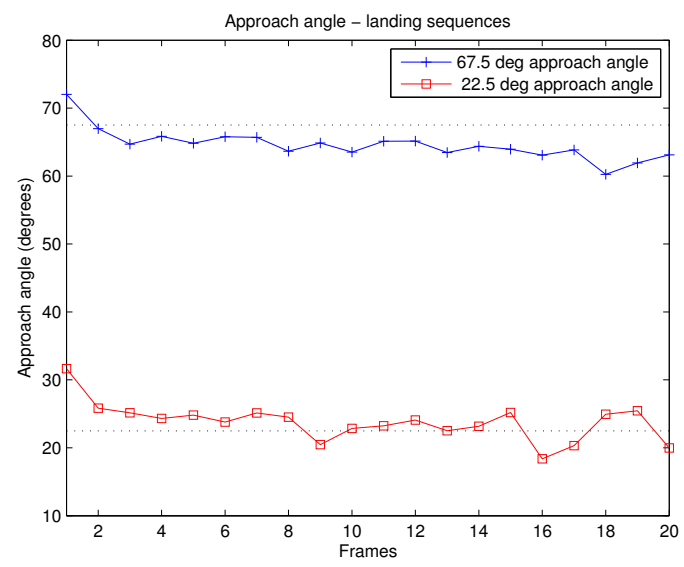

Fig. 4. Estimated angles of approach for landing sequences depicting a $22.5^{\circ}$ and $67.5^{\circ}$ approach. Dotted line indicates ground truth.

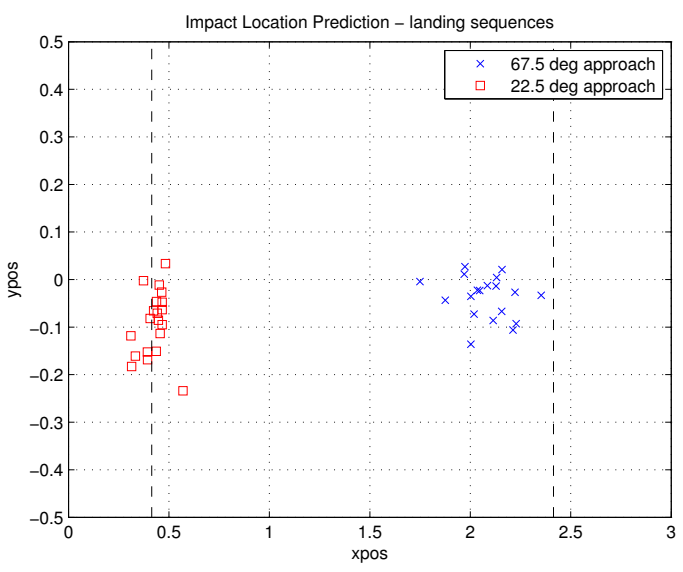

Fig. 5. Estimated location of impact for $22.5^{\circ}$ and $67.5^{\circ}$ landing sequences. The dashed line indicates ground truth.

of a camera descending towards a planar surface along a predetermined angle of approach, and at constant velocity. For this we assume the object of interest lies along the camera's viewing direction, and thus skip the segmentation phase. We tested the algorithm over sequences depicting a $22.5^{\circ}$ and $67.5^{\circ}$ approach. Figure 3 shows sample frames, and the central region used for estimating angle and location of impact.

Figure 4 shows results obtained for the estimated angle of approach of the surface in the central image patch. In both cases, a reasonable approximation to ground-truth is obtained. The $67.5^{\circ}$ approach deviates further from the ground truth, however, these estimates are more consistent than the $22.5^{\circ}$ approach. It should be noted that the angles of approach reported for both sequences are subject to some error due to the imprecise nature of the image construction process.

Figure 5 shows the estimated location of impact for each frame of both sequences. The origin of the coordinate system is the camera location. The location of impact is given in units of the estimated time-to-contact of the surface towards the camera. Thus, we expect the location to remain constant across 


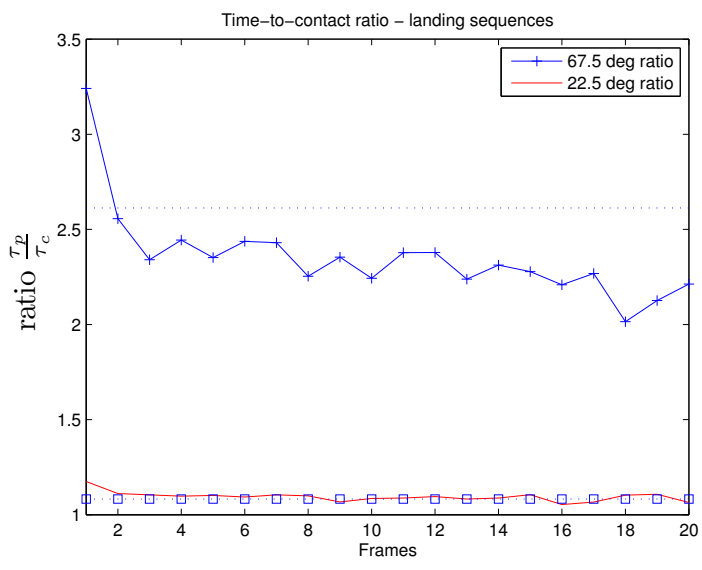

Fig. 6. The ratio of surface time-to-contact along the direction of object motion and the camera's central axis. Dashed line gives ground truth, as determined from the camera's known approach.

each sequence. It can be seen that the impact prediction is more accurate for the $22.5^{\circ}$ approach than the $67.5^{\circ}$ approach. However, both remain stable throughout the descent.

To assess the accuracy of time-to-contact estimates with the impact location, we compare the ratio of time-to-contact estimates obtained along the direction of motion, and along the camera's central axis. Accurate time-to-contact estimates should yield a constant ratio across the sequence. Figure 6 shows the time-to-contact ratio results. It is clear that high accuracy and consistency is achieved across the $22.5^{\circ}$ trial. Consistency and accuracy degrade for the $67.5^{\circ}$ trial, although in general, the estimated ratios remain stable.

\section{Live impact prediction test}

The full algorithm was implemented for real-time use with a stationary digital camera. The predicted impact point, and time-to-contact were both computed at frame rate, and displayed in an adjacent image. A supplementary video shows real-time footage of the system output during the experiment. Figures 7 and 8 show sample frames from the video output, showing both the detection of the incoming object (in the left image), and the predicted location of impact about the plane surrounding the camera (right image). Impact predictions for an object of interest are represented by rectangular markers, and are shown for all predictions prior to and including the current frame. Increasing brightness in impact point markers represents decreasing time-to-contact.

In both cases, early predictions appear to be outliers compared with later predictions. Accuracy of impact predictions also appear stronger for the open hand than for the closedfist, suggesting the approximate planar surface of the open hand yields better predictions. This is unsurprising given the algorithm assumes depth variation within the region of interest is zero. Depth variation across the closed fist is likely to have an additive effect to divergence, thereby biasing impact location prediction towards the viewer direction, as can be seen in later impact predictions for the closed-fist example.

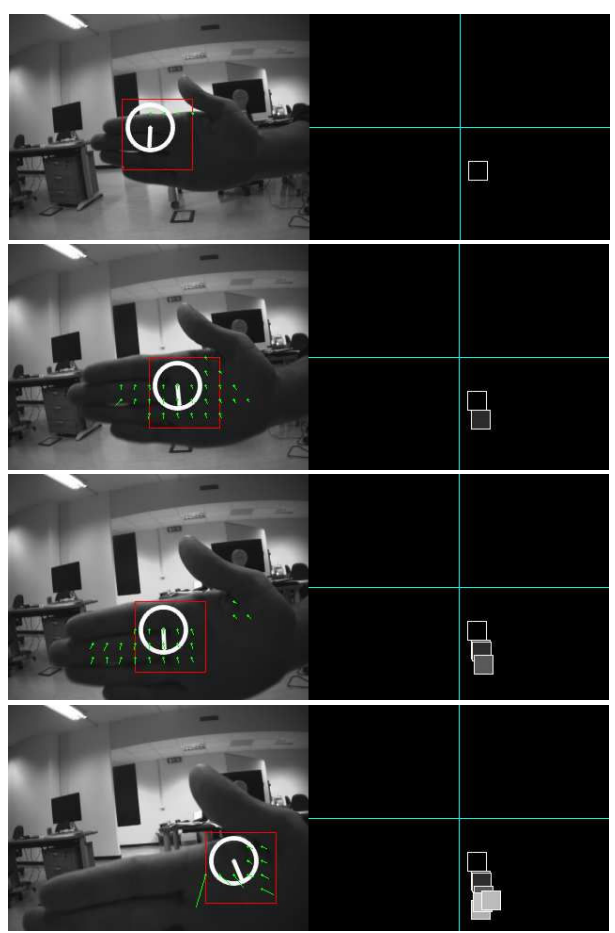

Fig. 7. Real-time impact predictions for an incoming open hand.

\section{Discussion}

In general, the proposed scheme exhibits stable and consistent performance, with degraded accuracy for significantly distant impact locations. Impact predictions for more eccentric trajectories appear to be biased towards the viewing direction. Notably, this bias is consistent with results reported from human trials, where it has been suggested this may be an evolved defensive adaptation to protect peri-personal space [21]. The bias observed here is most likely due to local deformation from depth change across non-fronto-parallel surfaces (e.g.the closed-fist). This will have an additive on the total divergence measured, creating a biasing towards the viewing axis.

Impact locations are expressed in temporal units, which may naturally be extended to support the visual control of object interception and avoidance. The temporal scale also provides a more accurate model of the velocity-determined variation of the receptive field depth of bimodal neurons. Faster moving objects are detected earlier, as observed in primate experiments [1].

\section{CONCLUSION}

We have presented preliminary work in developing a scheme for predicting the location of impact for an incoming object, based on the object's instantaneous pattern of optical flow. The scheme estimates the location of impact on a planar body, centred on the location of the camera. We achieve this by examining both the translational component of the object's optical flow, and the divergence. Results demonstrate workable accuracy over a range of approach angles, and its suitability 


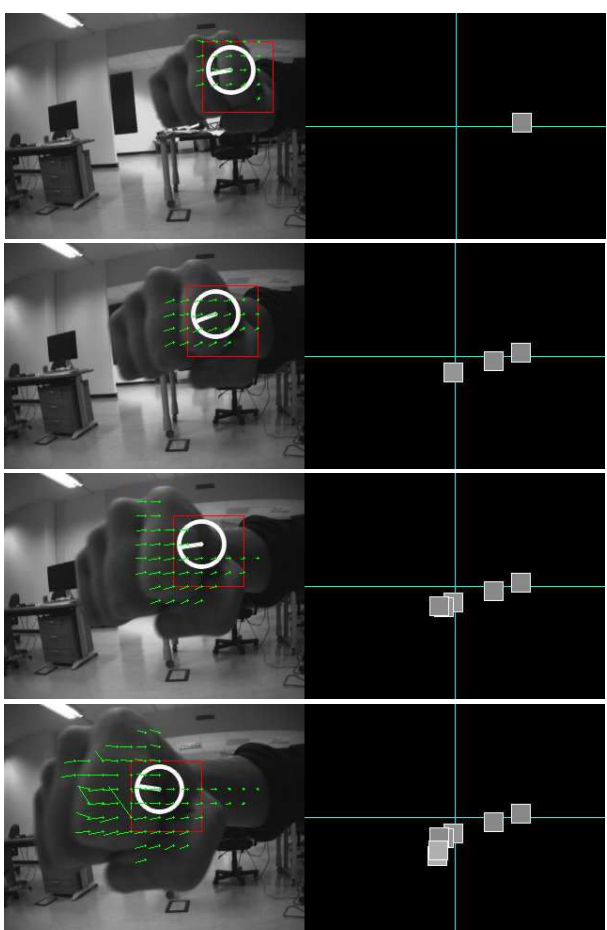

Fig. 8. Real-time impact predictions for an incoming closed fist.

for real-time use. While accuracy degrades for significantly distant impact points, results remain stable and consistent.

\section{A. Future work}

A more complete model of F4 neurons has to combine visual responses (similar to those presented in this paper) with tactile responses and potentially use this representation for controlling reaching. To this end, several building blocks have been developed already on the iCub platform. They include a complete skin that covers the robot's upper body [22] and fingertips [23] together with a flexible motor control system for planning reaching movements [24]. Clearly, merging vision with touch while maintaining a stable reference frame requires some extensions to the present method as noted above: e.g., moving cameras, learning, and moving body parts.

\section{ACKNOWLEDGEMENTS}

NICTA is funded by the Australian Government as represented by the Department of Broadband, Communications and the Digital Economy and the Australian Research Council through the ICT Centre of Excellence program. This work has been supported by the EU FP7 project CHRIS (grant no.215805) and RoboSKIN (grant no.231500).

\section{REFERENCES}

[1] L. Fogassi, V. Gallese, L. Fadiga, G. Luppino, M. Matelli, and G. Rizzolatti, "Coding of peripersonal space in inferior premotor cortex (area f4)," Journal of Neurophysiology, vol. 76, no. 1, pp. 141-157, July 1996.

[2] L. Fogassi, V. Gallese, G. di Pellegrino, L. Fadiga, M. Gentilucci, G. Luppino, M. Matelli, A. Pedotti, and G. Rizzolatti, "Space coding by premotor cortex," Experimental Brain Research, vol. 89, pp. 686-690, 1992.
[3] G. Rizzolatti, L. Fadiga, L. Fogassi, and V. Gallese, "The space around us," Science, vol. 277, pp. 190-191, 1997.

[4] M. S. Graziano, "Where is my arm? the relative role of vision and proprioception in the neuronal representation of limb position," Proceedings of the National Academy of Science, USA, vol. 96, pp. 10418-10421, August 1999.

[5] H. Li, "Two-view motion segmentation from linear programming relaxation," in Proceedings of the IEEE Conference on Computer Vision and Pattern Recognition (CVPR), 2007, pp. 1-8.

[6] R. Vidal and R. Hartley, "Motion segmentation with missing data using PowerFactorization and GPCA," in Proceedings of the IEEE Conference on Computer Vision and Pattern Recognition $(C V P R)$, June 2004, pp. II-310 - 316. [Online]. Available: http: //dx.doi.org/10.1109/CVPR.2004.162

[7] K. Yamaguchi, T. Kato, and Y. Ninomiya, "Vehicle ego-motion estimation and moving object detection using a monocular camera," in Proceedings of the International Conference on Pattern Recognition (ICPR), vol. 4, 0-0 2006, pp. 610-613.

[8] I. Cohen and G. Medioni, "Detecting and tracking moving objects in video from an airborne observer," in Proceedings of IEEE Image Understanding Workshop, 1998, pp. 217-22.

[9] A. Mitiche and H. Sekkati, "Optical flow 3D segmentation and interpretation: A variational method with active curve evolution and level sets," IEEE Transactions on Pattern Analysis and Machine Intelligence, vol. 28, no. 11, pp. 1818-1829, 2006.

[10] J. Weber and J. Malik, "Rigid body segmentation and shape description from dense optical flow under weak perspective," IEEE Transactions on Pattern Analysis and Machine Intelligence, vol. 19, no. 2, pp. 139-43, 1997.

[11] D. N. Lee, "A theory of visual control of braking based on information about time to collision," Perception, vol. 5, no. 4, pp. 437-59, 1976.

[12] R. C. Nelson and J. Y. Aloimonos, "Obstacle avoidance using flow field divergence," IEEE Transactions on Pattern Analysis and Machine Intelligence, vol. 11, no. 10, pp. 1102-6, 1989.

[13] N. Ancona and T. Poggio, "Optical flow from 1d correlation: Application to a simple time-to-crash detector," in Proceedings of the IEEE International Conference on Computer Vision (ICCV), 1993, pp. 209-14.

[14] R. Cipolla and A. Blake, "Image divergence and deformation from closed curves," International Journal of Robotics Research, vol. 16, no. 1, pp. 77-96, 1997.

[15] C. McCarthy, N. Barnes, and R. Mahony, "A robust strategy for a mobile robot using flow field divergence," IEEE Transactions on Robotics, vol. 24 , no. 4, pp. 832-842, 2008.

[16] J. S. Chahl, M. V. Srinivasan, and S. W. Zhang, "Landing strategies in honeybees and applications to uninhabited airborne vehicles," International Journal of Robotics Research, vol. 23, no. 2, pp. 101-110, 2004.

[17] J. J. Koenderink and A. J. van Doorn, "Local structure of movement parallax of the plane," Journal of the Optics Society of America, vol. 66, no. 7, pp. 717-723, 1976.

[18] M. Subbarao, "Bounds on time-to-collision and rotational component from first-order derivatives of image flow," in Computer Vision, Graphics and Image Processing, vol. 50, 1990, pp. $329-41$.

[19] T. Brodsky, C. Fermüller, and Y. Aloimonos, "Direction of motion fields are hardly ever ambiguous," International Journal of Computer Vision, vol. 26, no. 1, pp. 5-24, 1998.

[20] B. Lucas and T. Kanade, "An iterative image registration technique with an application to stereo vision." in Proceedings of DARPA Image Understanding Workshop, 1981, pp. 121-130.

[21] M. Neppi-Mòdona, D. Auclair, A. Sirigu, and J.-R. Duhamel, "Spatial coding of the predicted impact location of a looming object," Current Biology, vol. 14, pp. 1174-1180, 2004.

[22] G. Cannata, M. Maggiali, G. Metta, and G. Sandini, "An embedded artificial skin for humanoid robots," in International Conference on Multi-sensor Fusion and Integration, Seoul, Korea, August 20 - 22, 2008, 2008.

[23] A. Schmitz, M. Maggiali, L. Natale, B. Bonino, and G. Metta, "A tactile sensor for the fingertips of the humanoid robot icub," in IEEE/RSJ International Conference on Intelligent Robots and Systems Abbreviated Journal, Taipei, Taiwan, October 18-22, 2010, 2010.

[24] U. Pattacini, F. Nori, L. Natale, G. Metta, and G. Sandini, "An experimental evaluation of a novel minimum-jerk cartesian controller for humanoid robots," Taipei, Taiwan, October 18-22, 2010, 2010. 